www.jmscr.igmpublication.org

Impact Factor 5.244

Index Copernicus Value: 5.88

ISSN (e)-2347-176x ISSN (p) 2455-0450

crossref DOI:_http://dx.doi.org/10.18535/jmscr/v4i4.19

Journal Of Medical Science And Clinical Research

IGM Publication

An official Publication of IGM Publication

\title{
Comprehensive study of Foetal cases of Sirenomelia Sequence with their Embryological Correlations
}

\author{
Authors \\ Usha Rani Vanagondi ${ }^{*}$, Saritha. $\mathbf{S}^{2}$, Jwalaram Kumar Chaluvadi ${ }^{3}$, \\ Gayathri. $\mathbf{P}^{4}$, Nagajyothi. $\mathrm{D}^{5}$ \\ ${ }^{1}$ Associate Professor, Department of Anatomy, Rajiv Gandhi Institute of Medical Sciences(RIMS), \\ Ongole, Prakasam District, Andhra Pradesh, INDIA \\ Email: v.usharani53@yahoo.com,+919000620012 \\ ${ }^{2}$ Professor\& HOD, Kamineni Academy of Medical Sciences \& R.C, L. B. Nagar, Hyderabad, \\ Telangana, India \\ Email: Kmr.saritha@gmail.com \\ ${ }^{3}$ Assistant Professor, Department of Anatomy, Rajiv Gandhi Institute of Medical Sciences(RIMS), \\ Ongole, Prakasam District, Andhra Pradesh, INDIA \\ Email:drjwalaram@gmail.com \\ ${ }^{4}$ Assistant Professor, Kamineni Academy of Medical Sciences \& R.C, L. B. Nagar, Hyderabad, \\ Telangana, India \\ Email: dr.gayatri.p@gmail.com \\ ${ }^{5}$ Assistant Professor, Kamineni Academy of Medical Sciences \& R.C, L. B. Nagar, Hyderabad, \\ Telangana, India \\ Email: jyothidandua@gmail.com \\ *Correspondence Author
}

Dr V. Usha Rani

Associate Professor, Department of Anatomy, Rajiv Gandhi Institute of Medical Sciences(RIMS), Ongole,

Prakasam District, Andhra Pradesh, INDIA

Email:v.usharani53@yahoo.com,+919000620012

\begin{abstract}
ABSTRCT
Comprehensive study of congenital anomaly "Sirenomelia" of the fetuses is a rare malformation which is incompatible with life. This is very rare anomaly and it is heart wrenching to hear such cases. The sequence was originally described by Rocheus in 1542 and Palfyn in 1953 and named after the mythical Greek sirens. Sirenomelia sequence is also known as Mermaid Syndrome, a Greek Mythology fact that it has two fused lower limbs look like mermaid's tail. Sirenomelia was formerly thought to be an extreme case of Caudal Regression Syndrome (CRS); but CRS is now considered to be caused by an anomaly of lower spinal trunk ending (Frog-like). It is encompasses by a wide range of anomalies including partial or complete agenesis of thoraco-lumbosacral spine \& pelvic deformities. Sirenomelia may be caused by abnormalities in blastogenesis that affect multiple midline primordial structures during the final stages of Gastrulation at the caudal eminence. This leads to insufficient migration and differentiation of mesoderm which is responsible for the array of defects in caudal region and it also affects the distribution of blood to the caudal region of the fetus. Approximately 300 cases have been reported in the literature with male:
\end{abstract}


female ratio being 3:1. These infants usually do not survive for more than 24 hours. Very few survive even after surgery.

Aim: Ultra sound scan may be useful in early antenatal detection of this anomaly. In view of bad prognosis earlier intrauterine diagnosis allows less traumatic therapeutic abortion minimizing termination of pregnancy at advanced gestation.

Observation \& Results: We observed two cases of Sirenomelia sequence.

First case was 25 years old primigravida at 24 weeks of gestation was admitted to the hospital. Prenatal ultrasound demonstrated severe Oligohydramnios and fusion of the lower limbs suggesting the diagnosis of Sirenomelia, pregnancy was terminated.

Second case was also 25 years old primigravida gave birth to still born full term baby, sex could not be identified. The specimen was collected from private nursing home and the diagnosed as Sirenomelia.

Both the cases had no relevant history of consanguineous marriage or tobacco smoking or drugs taking etc. Both the fetuses were submitted for detailed autopsy in anatomy dissection hall. The findings, with brief review of the literature, discussion of etio-pathogenesis and embryological correlation were presented in this article

Key words: Sirenomelia, Caudal Regression Syndrome (CRS), Vascular steal theory and Teratogenesis.

\section{Introduction}

The condition Sirenomelia sequence occurs commonly in males, with a sex ratio of $2.7: 1$ (Murphy et al, 1992) ${ }^{\mathbf{1}}$ Sirenomelia is not associated with chromosomal aneuploidy. Sirenomelia sequence or Symelia or Mermaid syndrome" is a rare lethal congenital anomaly characterized by fusion, rotation, hypotrophy or atrophy of the lower limbs, single umbilical artery and severe urogenital abnormalities leading to oligohydramnios. It is associated with renal agenesis, absence of both internal and external genitalia, with agenesis or hypoplasia of diverse organs and Monopedia. This rare malformation sequence characterized by fusion of the lower limbs, the spectrum of lower limb anomalies ranges from simple fusion of soft tissues to the presence of single rudimentary limb. (Lutz et al., 2004). ${ }^{2}$ There is no consistent data for the incidence of Sirenomelia. It is estimated to be between 1.1-4.2 cases per 100,000 births (Horikoshi et al 2005) ${ }^{3}$ and reported as 0.8 to 4.2 in 100,000 births (Stanton 2003) ${ }^{4}$ Most of cases are sporadic and have very little chance of recurring in next pregnancy. The aim is stressed on fact a compulsory antenatal ultrasound scanning for fetal anomalies are necessary for prompt diagnosis.

\section{Two Case Reports \\ First Case Report}

A 25 year old female with 24- 25 weeks of gestation was admitted to the hospital and on ultra sound scan was diagnosed to have oligohydramnios and lack of fetal movements. No $\mathrm{h} / \mathrm{o}$ diabetes or any teratogenic affects. The fetus demonstrated with fusion of lower limbs. The diagnosis of Sirenomelia was made. At the level of kidneys, bilateral enlarged polycystic structure was identified and other multiple interesting anomalies were seen. Because of incompatibility with postnatal life, pregnancy was terminated with the consent of the parents. Detailed fetal autopsy was done in anatomy dissection hall and findings were presented in the report. Since there were fused lower limbs seen, the fetus was subjected to radiological examination. The affected fetus presented with fused lower limbs and sacral agenesis.

Gross external features. (Foetus A -Fig1 \&2).

1. Upper limb length was normal measured about: $10 \mathrm{~cm}$ and there was discolorationof right upper limb.

2. Lower limbs were fused \& forming single lower limb whose length was about $7.5 \mathrm{~cm}$.

3. The thighs and legs were fused with ill-defined muscles. The two feet were attached at the ankle joint and rotated laterally, abducted and everted 
4. The external genitalia was absent and it was represented as small flap and absence of anal orifice

(Imperforate anus)

\section{Internal features}

On dissection placenta, heart was unremarkable and brain with absence of sulci.

1. Abdominal anomalies: The abdominal cavity includes multiple anomalies. It had a small liver with agenesis of gallbladder, annular pancreas around duodenum. The stomach and spleen were normal. The intestines were malrotated with small intestine on the right and large intestine on left side. The blind end of sigmoid colon opens into the cloaca and there was an atresia of anorectal canal. (Foetus A; Fig-3)

2. Urogenital anomalies include bilateral polycystic kidneys, two ureters opening in the cloaca.

The bladder and urethra were absent. There was small gonad on the left side of the abdomen. Cloaca receives the two openings of the ureters and sigmoid colon. (Foetus A;Fig-4)

3. Vascular anomalies: Abdominal aorta continuous as superior mesenteric artery (SMA) the steal vessel which continuous as single umbilical artery or aberrant umbilical artery (SUA). Further there was caudal tapering of abdominal aorta as hypoplastic aorta and it ended at the pelvic brim.

The inferior mesenteric artery (IMA) arose from abdominal aorta above the origin of superior MA. (Foetus A; Fig-5)

\section{Radiology findings: (Foetus -A; Fig-6):}

1. Upper Limb Bones Normal

2. Lower Limb Bones: Hip Bones, Femora, Tibiae, Fibulae were present and were normal \& feet rotated with Sacral agenesis.

5. Umbilical cord showing single Umbilical artery and vein with microscopic images. (Foetus -A; Fig-7)

6. Microscopic picture of single gonad (primitive) (Foetus -A; Fig-8)
7. CT scan- (Foetus A, Fig-8): Brain showing absence of Sulci, parenchymal hypodensity, Hypodense cortical sinus secondary to stasis of blood

\section{Impression}

Soft tissue fusion in the region of thigh, leg up to the ankle region. Both feet are separated and rotated lateral. It was diagnosed as mild mermaid syndrome, with sacral agenesis and fusion restricted to skin and soft tissue

\section{Second Case Report}

Second case was also 25 years old primigravida gave birth to still born full term baby, sex could not be identified .The specimen was collected from private nursing home and the diagnosis was of Sirenomelia. No h/o diabetes or any teratogenic affects. The full term fetus small for gestational age and of undetermined sex was born by spontaneous vaginal delivery. The infant was demonstrated with fusion of lower extremities and showed absence of external genitalia. Detailed fetal autopsy was done in anatomy dissection hall and findings were presented in the report. Since there was a fused lower limb seen, the fetus was subjected to radiological examination

External features: (Foetus B; Fig 1\& 2)

1. The eyes are amphibian-like and the ears are floppy and low set.

2. The Upper extremity normal with abnormal fingers.

3. Only one left lower limb and it was not completely formed.

4. Absence of external genitalia and No urethra and anal openings.

\section{Internal features}

Fetal autopsy was done and following features were observed.

The Placenta no remarkable findings and Brain with absence of sulci.

1. Thorax: The heart and thymus were normal and lungs were hypoplastic. (Foetus B; Fig-3) 
2. Abdominal cavity showed multiple anomalies. The Gut rotation was complete. The Stomach, small intestine, liver and spleen were normal. The Caecum and Ascending colon distended with meconium and Rectum ended blindly. (Foetus B; Fig-4)

3. Urogenital anomalies: There was complete renal agenesis, No kidneys, Ureters and Bladder. Gonad (single) was seen in inguinal canal of right side. (Foetus B; Fig-5)

4 Vascular anomalies: Blood vessels of abdomen. (Foetus B; Fig-6\& 7)

Abdominal Aorta has no division of Common Iliac arteries and complete absence of External \& Internal iliac arteries. There was a small branch from abdominal aorta entering into the left lower limb. Superior\& Inferior mesenteric arteries were ill defined A single umbilical artery was present, which was in direct continuity with the abdominal aorta.

5. Umbilical cord showing single Umbilical artery and vein (Foetus B; Fig-8)

6. Microscopic structure of Testis \& Epididymis (Foetus B; Fig-9)

\section{Foetus - A}

7. Microscopic structure of Thymus (Foetus B; Fig-10)

8. X-ray findings: (Foetus B; Fig-11\&2)

Vertebral anomalies: Clefting seen in $T_{3}, T_{5}$ and $\mathrm{T}_{6}$, Scoliosis - convexity to left Spina bifida at lumbar level. Distal sacral segment not seen.

Partial agenesis of sacrum present.

Limb defects:

Right hand: Polydactyly and Syndactyly of right thumb

Lower limbs: Single left lower limb was present, no fusion.

One femur, one tibia, no fibula

No tarsal bones \& only small (little toe) metatarsal present

\section{Diagnosis: SIRENOMELIA}

9. CT scan Brain: Sulci absent, parenchymal hypodensity.

Hypodense cortical sinus secondary to stasis of blood

Limbs: One left lower limb with femur, tibia and no fibula.

Impression: Sirenomelia

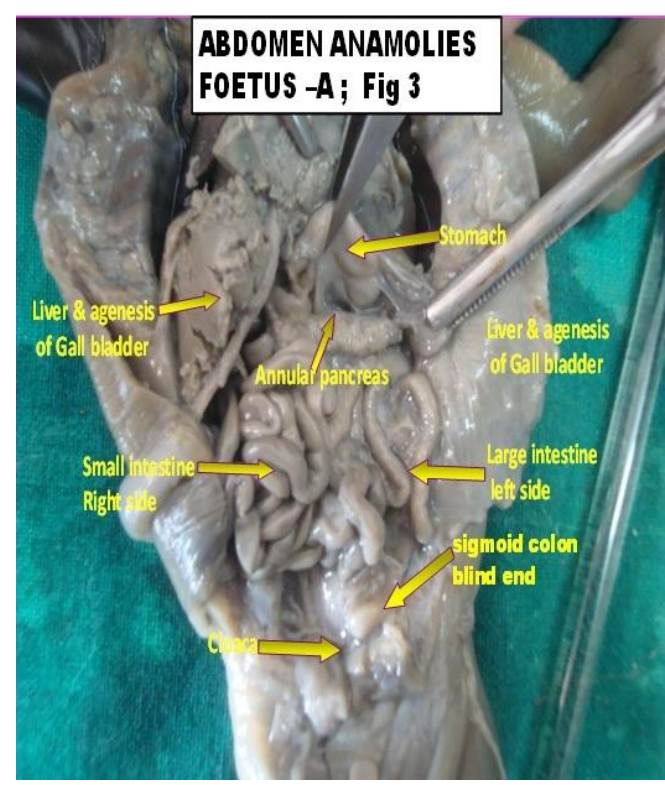




\section{JMSCR Vol||04||Issue||04||Page 10074-10084||April}
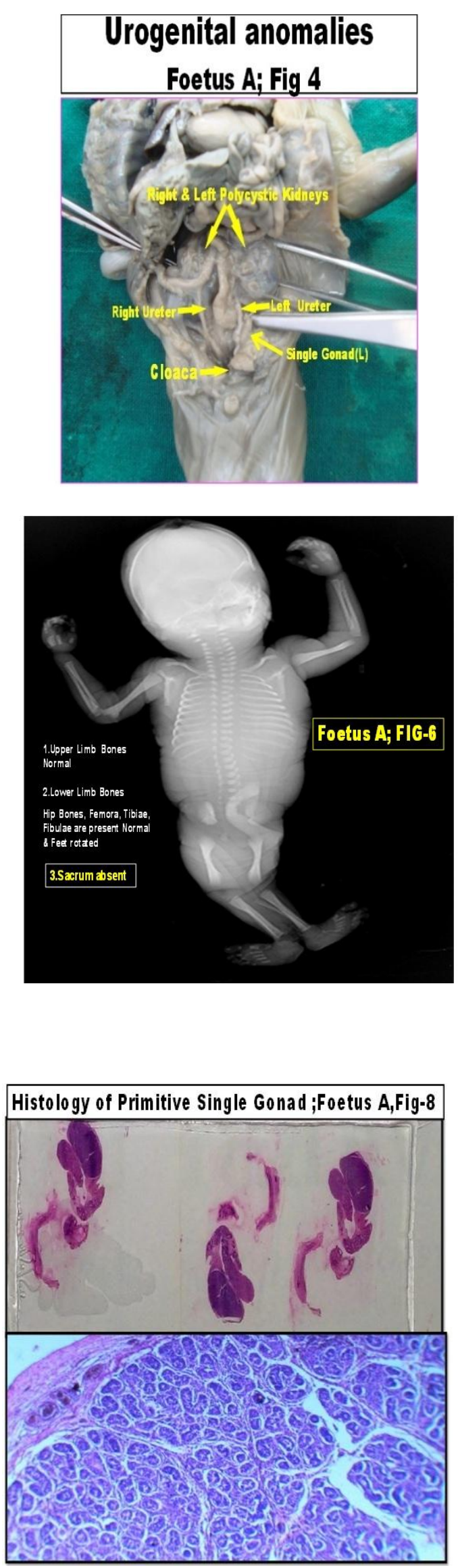
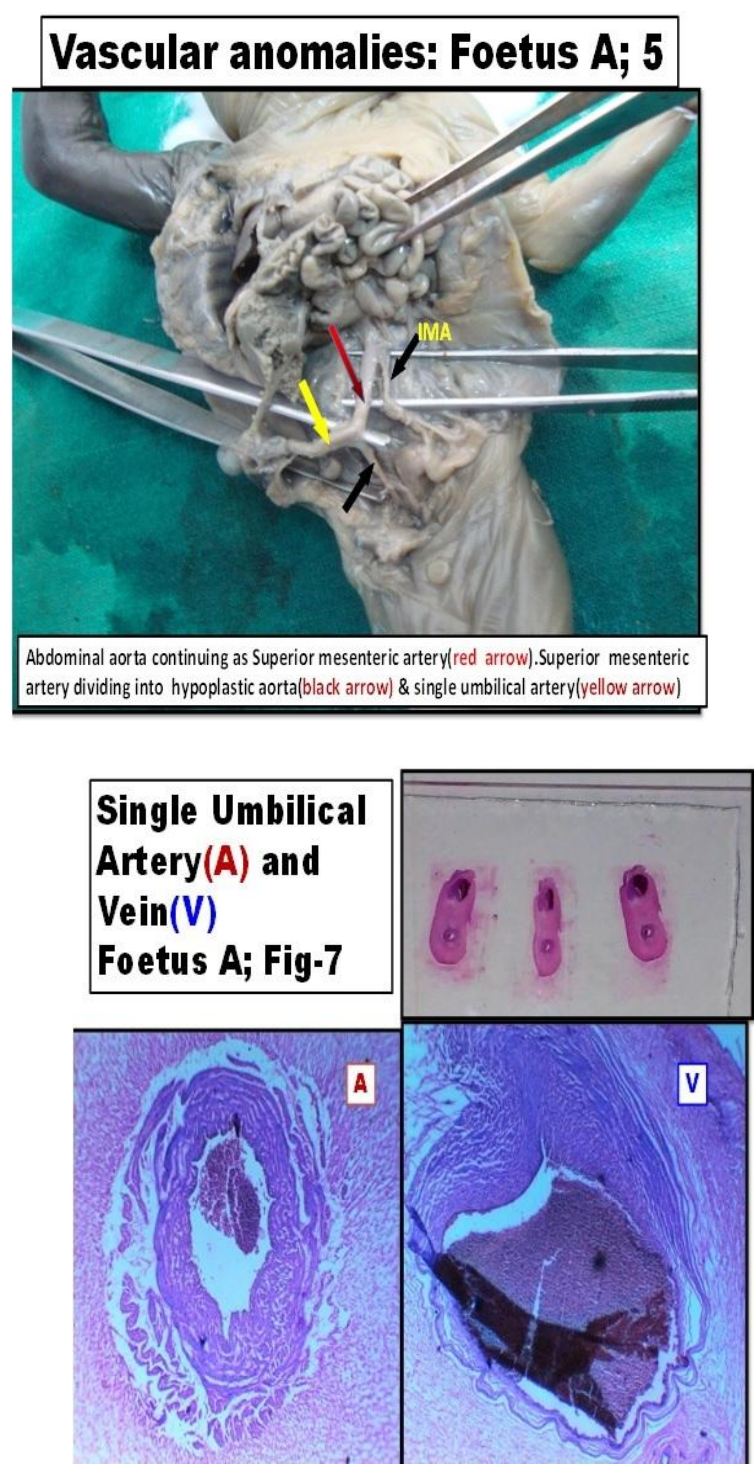

\section{CT scan- Foetus A,Fig- 9}

Brain: Sulci absent, parenchymal hypodensity, hypodense cortical sinus secondary to stasis blood.

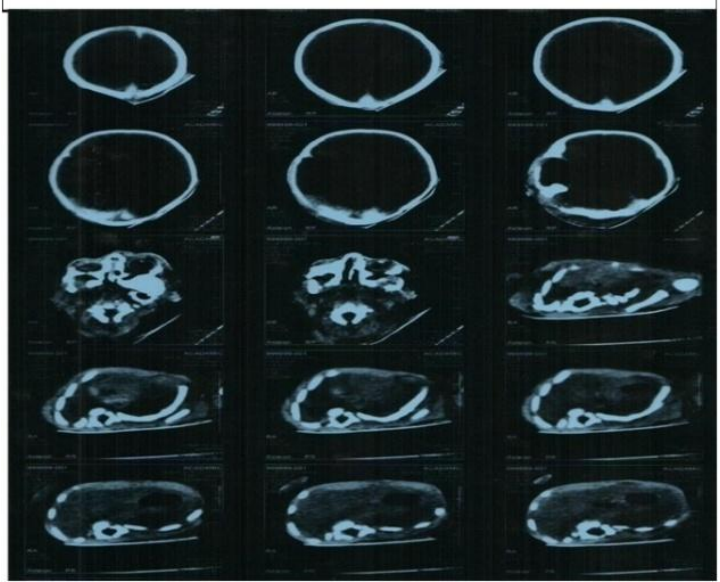




\section{JMSCR Vol||04||Issue||04||Page 10074-10084||April}

Foetus - B
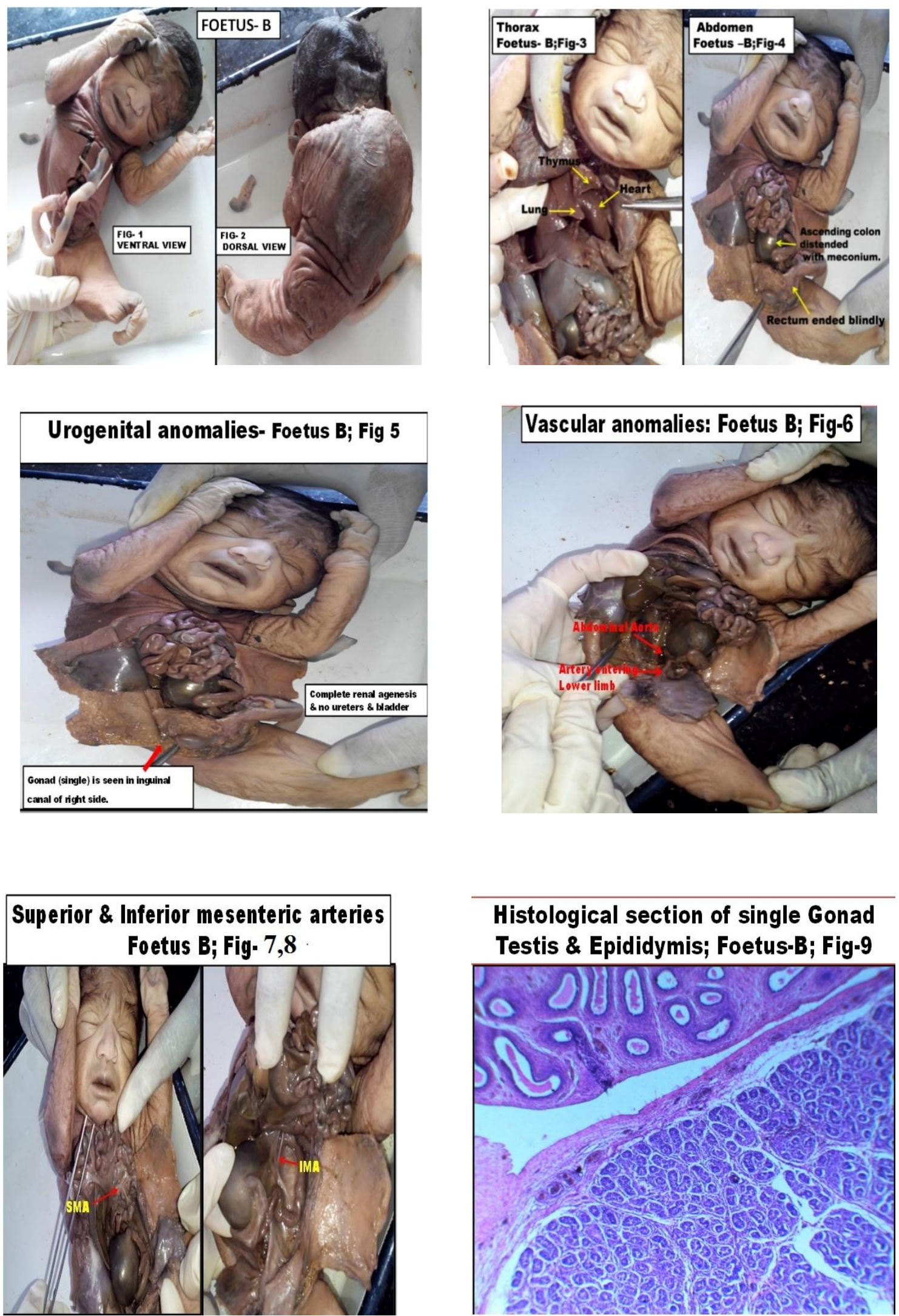

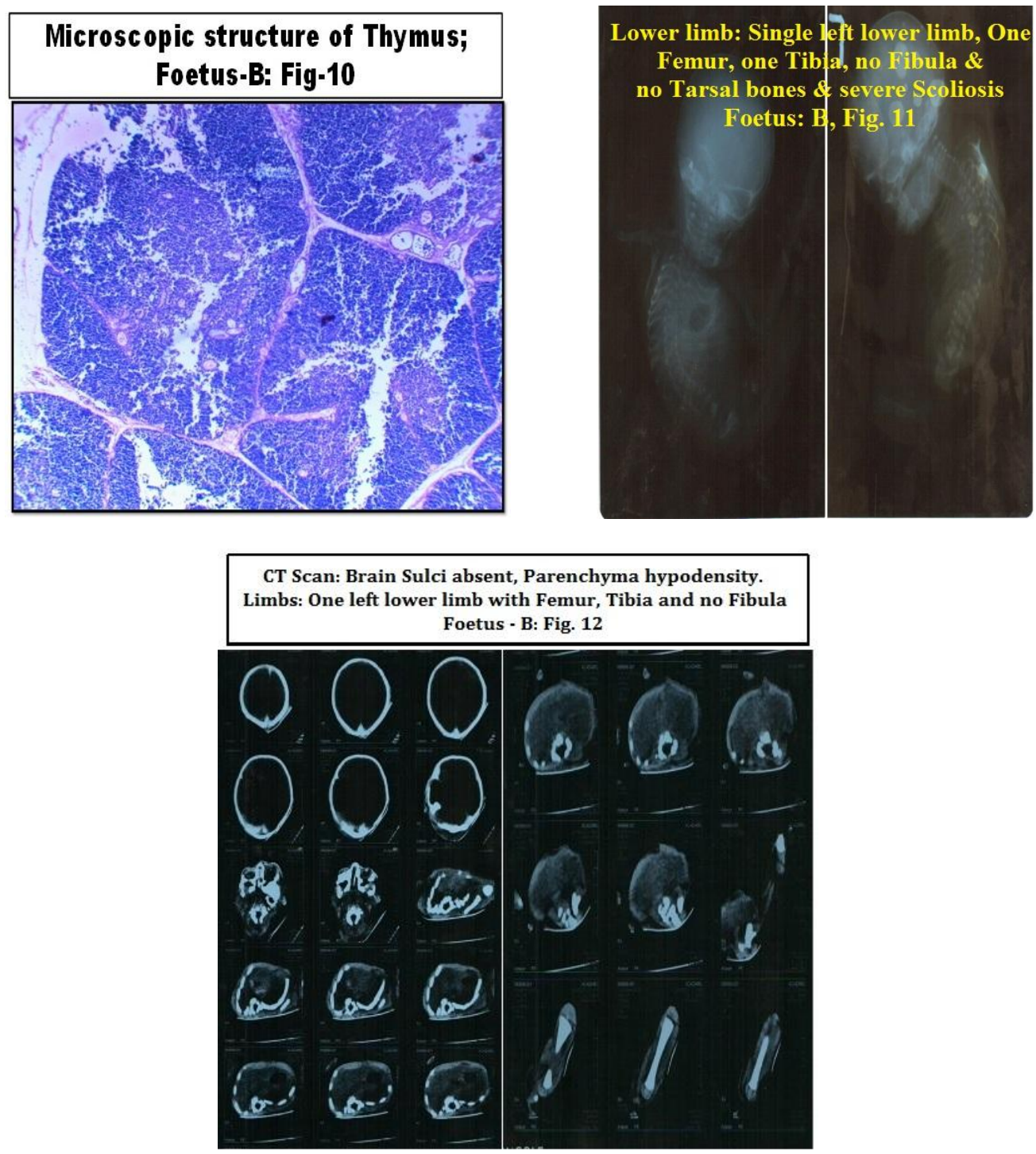

\section{Discussion}

Sirenomelia or the Mermaid syndrome is a rare congenital dysmorphic syndrome of the lower body segments and with fusion of the lower limbs. The first reported case of mermaid syndrome was in the $16^{\text {th }}$ century. The etiology of Sirenomelia is unclear though it is well known that the embryological injury occurs between 28 and 32 days of intra uterine life. Sirenomelia was formerly thought to be an extreme case of Caudal Regression Syndrome (CRS); however, it was reclassified to be considered a separate condition. Sirenomelia is a lethal condition characterized by fusion of lower limbs, single umbilical artery and severe malformations of lower GIT and Urogenital systems associated with renal agenesis (Mermaid Tail). On the contrary CRS is also rare disorder associated with two umbilical arteries and non fusion of lower limbs. It is characterized by various degrees of developmental failure involving legs, lumbar, sacral and coccygeal vertebra, spine including sacral agenesis and corresponding segments of spinal cord are also involved due to defect in neuralisation followed by motor and sensory deficits. The defects predominantly involving the lower portion of the body, mainly in lower spine end of fetus (frog like). Sirenomelia is associated with Oligohydramnios and Caudal Regression Syndrome is associated with polyhydramnios and strongly associated with maternal diabetes and therefore they are two different entities. Sirenomelia is thought to result from "Vascular Steal Syndrome". Diversion of blood flow away from the caudal portion of the embryo through the abdominal umbilical artery/“vascular steal" has been proposed as the primary mechanism leading to Sirenomelia. The presence of single umbilical 
artery $\&$ associated with renal agenesis and fusion of lower limbs, not compatible with life. In contrast, CRS is hypothesized to arise from primary deficiency of caudal mesoderm. A teratogenic event during the gastrulation stage, the $3^{\text {rd }}$ gestational week, may interfere with the formation of notochord, resulting in abnormal development of the caudal structures .The presence of two umbilical arteries, non lethal renal anomalies and divided lower limbs, CRS relatively compatible with life.

\section{Vascular malformations}

Heifetezet et al 1984 \& Stevenson et al. reported that the vascular abnormalities in Sirenomelia. They explained diversion of blood away from the caudal region of the embryo through the abdominal umbilical artery "A vascular steal" has been leading etiological factor to Sirenomelia. Human umbilical cord normally has two umbilical arteries carrying deoxygenated blood from the fetus to placenta $\&$ single umbilical vein carrying oxygenated blood to the fetus. However invariably Sirenomelia fetuses exhibit a single umbilical artery $^{\mathbf{5}, 6}$. Heifetezet et al 1984 \& Stevenson et al considered the presence of single umbilical artery (SUA) of vitalline origin as characteristic and pathognomonic of Sirenomelia and forms differential diagnosis of other malformations such as caudal dysgenesis or CRS. This SUA branches from the abdominal aorta at a quite high in the abdomen, thereafter the Aorta narrows and lacks number of branches supplying kidneys, large intestine \& genitalia. SUA steals the blood from the structures below it- Vascular steal hypothesis (Martinez-Frias etal) ${ }^{7}$. Recent studies have shown that vascular disruption precedes caudal dysgenesis in the mouse ${ }^{8}$

Three pathogenic theories have been proposed to explain this malformation. (Stevenson et al) ${ }^{\mathbf{6}, 7,8}$

1. A pressure theory (Oligohydramnios and intrauterine force acts on the tail of the embryo may impede normal rotation of limb buds).
2. Primary failure in the development of caudal somities that leads to defective development of lower parts of the embryo.

3. A lack of nutritional support of the caudal region of the embryo. (Vascular steal theory)

The present study both Sirenomelia fetuses had single umbilical Artery (SUA) associated with severe Oligohydramnios leading to ill defined or agenesis caudal organs.

\section{Hind limb fusion types:}

The classification of Sirenomelia infants on presence or absence of bones within the lower limb was made by Stocker and Heifetz. ${ }^{9}$ in1987 into 7 types. Stocker's more detail classification into7 Sub types according to the fused bones.

Type - I : Paired femora, Tibiae and Fibulae. Type - II : Paired femora, Tibiae \& single fused fibula.

Type - III: Paired femora, Tibiae \& absent fibula. Type - IV: Partially fused femora, tibiae \& single fibula.

Type - V: Partially fused femora, tibiae \& absent fibulae.

Type - VI: A single femur \& Tibia absent fibulae. Type - VII : A single femur and absent Tibiae and fibulae

Type I to Type VII mainly according to presence of skeletal elements in the thigh \& leg. Type I is mildest form, all bones in the fused limbs are present\& fusion affecting only soft tissues. In Type VII is most severe form, only single bone is present. In Type VI has single femur, Tibia \& absent fibulae.

Our first index case was 24 weeks fetus belongs Type I \& Second index case was full term still born fetus belongs Type VI. Both the fetuses multiple internal anomalies and associated with sacral agenesis.

Sarpong and Heading $1992^{10}$ classified the Sirenomelia syndrome into three types which is known as Sirtoris's classification.

SIRTORIS'S classification into 3 types according to the Number of feet. (Sarpong et al in 1992) ${ }^{\mathbf{1 0}}$ 
1. Sympus Apus [Absence of feet, only one tibia and one femur.]

2. Sympus Unipus [Presence of only one foot, two femora, two tibiae, two fibulae]

3.Sympus Dipus [Presence of both feet and two fused legs] fused appearance is of a flipper or fins. Accordingly the present report the clinical and anatomical features consistent with Sirenomelia dipus \& apus. Fetus A belongs to Sympus dipus with malrotated feet and associated with multiple internal anomalies \&Fetus B belongs to, Sympus apus ill formed foot also associated with multiple internal anomalies Visceral malformations.

Lutz et al., $2004^{2}$ stated that all human Sirenomelia fetuses are characterized by fusion of lower limbs with sacral agenesis and other anomalies like imperforate anus, colonic atresia and rectal atresia, renal agenesis and absent bladder. Usual gonads are present and external genitalia absent.

Duhamel et al in $1961^{11}$ also mentioned described the spectrum of visceral anomalies of the mermaid syndrome. He described imperforate anus, absence of genitalia (expect for gonads) renal agenesis, gastrointestinal anomalies including vascular anomalies like aberrant single umbilical artery. This anomaly is a manifestation of the mermaid syndrome.

The present two cases had all the above features mentioned by Lutz et al and Duhamel et al except for no renal agenesis in Fetus $\mathrm{A}$ and it presented with polycystic kidneys and ureters opening in to cloaca. Cloaca also receives the blind sigmoid colon and Fetus B was associated with renal and urinary bladder agenesis.

Kallen et al 1992 \& Goodlow et al $1998^{\mathbf{1 2}, 13}$ stated that malformation of urinary tract is consistently associated with genital anomalies affecting external genitalia which were either absent or represented as indistinct tag of tissue whereas gonads may be present.

In the current study both the fetuses malformations of urinary system, external genitalia represented as indistinct tag of tissue in
Foetus A \& Foetus B had no external genitalia. Both the fetuses had single gonad in the abdomen. In 1980 Gardner and Breuer ${ }^{14}$ proposed a theory of a neural tube over distension in the caudal area may lead to a roof plate expansion of the tube leading to lateral rotation of mesoderm by $180^{\circ}$. This results in fusion of the lower limb buds followed by closing off the midline primitive gut and urethra.

In present study the second fetus Spina bifida in lumbar region with absence of sulci in the Brain and Foetus A also had absence of sulci in the Brain with no spinal defects.

\section{Other malformations associated with Sirenomelia}

According to Stocker and Heifetz. ${ }^{9}$ in1987 \& Kjaer et al. $2003^{\mathbf{1 5}}$ other important commonly observed anomalies in mermaid syndrome are lumbosacral \& pelvic anomalies including sacral agenesis and hemivertebrae with also corresponding anomalies of central nervous system. Our both fetuses showed sacral \& coccygeal agenesis and Foetus B also had Spina bifida in lumbar region and Scoliosis with left side convexity.

Maternal teratogenesis associated with Sirenomelia has been described with maternal diabetes, cocaine or snuff exposure. In the monozygotic twins; the incidence is 150 times greater than in singleton. (Vander Zalen-Sprock et al, 1995; Di Lorenzo et al., 1991) ${ }^{\mathbf{1 6} \& 17}$.

Maternal diabetes is considered as causative environmental factor for Caudal Regression Syndrome (Caudal dysgenesis) because 10-15\% of affected children have diabetic mothers. However this association remains controversial for Sirenomelia because $0.5-3.7 \%$ of reported cases has diabetic mothers. (Stocker and Heifetz et. al $^{9}$ in1987; Ducan\&Sharpiro $1993^{18} \quad$ \& Duesterhoeft et.al $2007^{19}$ )

Currently there is no serum marker to diagnose Sirenomelia. Prenatal ultrasonography as early as 13 weeks of pregnancy can detect gross structural anomalies. So if diagnosed earlier the alternative 
of termination of pregnancy can be safely advised to the mother. Similarly proper control of blood glucose level in a diabetic mother may prevent the occurrence of Sirenomelia (Schiesser M et.al 2003) ${ }^{\mathbf{2 0}}$. Absence of chromosomal abnormalities and familial inheritance has been noted in almost all cases. Karyotyping of parents was normal as reported by number of researchers. This was done in the parents of both the fetuses and found normal. The evidences have shown that Sirenomelia associated with Oligohydromnias $0.5-3.7 \%$ of reported cases have diabetic mothers and Caudal regression Syndrome associated with polyhydromnias $10-15 \%$ of affected children have diabetic mothers. and therefore they are two different entities ${ }^{21}$.

Diversion of blood flow away from the caudal portion of the embryo through the abdominal umbilical artery/"vascular steal" has been proposed as the primary mechanism leading to Sirenomelia. In contrast, CRS is hypothesized to arise from a primary defect of the caudal mesoderm. ${ }^{22,23 \& 24}$

New reproductive technologies like Intra Cytoplasmic Sperm Injection(ICSI) was described to be associated with Sirenomelia. ${ }^{25}$

To conclude the precise etiology of the present two cases were labeled as rare variant of Sirenomelia and not Caudal regression syndrome of unknown etiology. Both the fetuses presented with Single umbilical artery \& renal anomalies which not compatible with life. However etiology, still remains unknown as both the mothers were non diabetic and not exposed to any teratogenic agents probably Vascular steal theory holds good.

\section{Conclusions}

The prognosis of Sirenomelia or mermaid syndrome represents a rare and fatal congenital malformation that is incompatible with life. Survival is extremely rare due to multiple agenesis or hypoplastic viscera associated with fused lower limbs. Early ultrasound in the first trimester may be useful in antenatal detection of this anomaly. In view of the bad prognosis earlier intra-uterine diagnosis allows less traumatic therapeutic abortion. Although in our both the Sirenomelia fetuses, the mothers did not have any evidence of any exposure to teratogenic factors, the couples should be counselled about early screening in subsequent pregnancy.

\section{References}

1. Murphy, J.J., G.C.Fraser and G.K. Blair, Sirenomelia: Case of the surviving mermaid. 1992; J. Pediatr. Surg., 27: 1265-12682.

2. Lutz, B.J.Meyrat, J.P.Guignard and J.Hohfeld, Mermaid syndrome: virtually no hope for survival. 2004; Pediatr. Surg.Int, 20: 559-561.

3. Horikoshi, T., A.Kikuchi, M. Tatematsu, Y.Matsumoto, A.Hayashi and N.Unno, Two cases of a fetus with Sirenomelia sequence, 2005; Congenit. Anom. 45: 9395.

4. Stanton, M.P., E.C. Pennington and J.M.Hutson, A surviving infant with Sirenomelia (Mermaid syndrome) associated with absent bladder, 2003; J. Pediatr. Surg., 38:1266-1268.

5. Heifetz S.A. Asingle umbilical artery.A statistical analysis of 277 autopsy cases and review of literature. Perspcept.Pediatr.Patho.1984:8:345-378

6. Stevenson RE. Jones KL, Phelan MC et al: Vascular Steal: the pathogenetic mechanism producing Sirenomelia and associated defects of the viscera and soft tissues, 1986; Pediatrics 78:451-7

7. Martinez-Frias M.L;BermejoE.RodriguesPinillaE.PrietoD.Does, a single umbilical artery predict any congenital defect? Clinical -Epidemiological analysis of a large consecutive series of malformed infants. Am.J.Med.Genet 2008;146A:1525

8. Padamanabhan R, Naruse I, Shiota K. Caudal regression in staged human 
embryos: Carnegie stages 16-23. Am J Med Genet. 1999;87:115-27. [PubMed

9. Stocker JT, Heifetz SA. Sirenomelia.A morphological study of 33 cases and review of the literature. Perspect Pediatr Pathol. 1987; 10:7-5.

10. Sarpong. And V. Headings, Sirenomelia accompanying exposure of embryo to cocaine, 1992; South. Med. J., 85: 545-547

11. Duhamel B. From the Mermaid to Anal Imperforation: The Syndrome of Caudal Regression. Arch Dis child .1961 Apr; 36 (186):152-155

12. Kallen B.; CastillaE. E.Lancaster P.A. Mutchinick O.KnudsenL.B.MartinezFriasM.L. Mastriocovo. P.Robert E. The cyclops and mermaid: epiemiological study of two types of renal formation. J.Med.Genet 1992;29:30-35

13. GoodlowO.G.SibleyR.I.Allen B.G: KamandaW.S.GullateeA.C.Rayfield W.C. Sirenmelia: mermaid syndrome.J.Natl .Med.Assoc. 1988;80:343 -346

14. Gardner NJ, Breuer AC. Anomalies of heart, spleen, kidneys, gut and limbs may result from an overdistended neural tube : A hypothesis. Pediatrics 1980; 65:508-514

15. KjaerK.W.KeelingJ.W.OpotzI.M.GilbratB arnessE.Hartling B..F;KjaerI.Sirenomelia sequence according to the distance between the first sacral vertebra \& the Ilia.Am.J.Med.Genet.2003:120A:503-508.

16. Van Zalen-Sprock, M.M., J.M. Van Vugt, J.J. Van Der Harten and H.P. Van Geijn, Early second-trimester diagnosis of Sirenomelia, 1995; Prenat. Diagn. 15: 171177

17. Di Lorenzo, M., M.L. Brandt and A. Veilleux, Sirenomelia in an identical twin: A case report, 1991; J. Pediatr. Surg., 26: 1334-1336
18. Ducan P.A.,ShapiroL.R.Interralationship of hemifacialmicrosomia-VATER,VATER and sirenomeliapheto types.Am. J.Med. Genet 1993; 47:75-84.

19. Duesterhoeft, S.M., L.M. Ernest, J.R. Siebert and R.P. Kapur, Five cases of caudal regression with an aberrant abdominal umbilical artery: Further support for a caudal regressionSirenomelia spectrum,2007; Am. J. Med. Genet. A., 143A: 3175-3184

20. Schiesser M, Holzgreve W, Lapaire O, Willi N, Luthi H, Lopez R, et al. Sirenomelia, the mermaid syndromedetection in first trimester. PrenatDiagn. 2003;23:493-5. [PubMed

21. Das BB, Rajegowda BK, Bainbridge R, Giampietro PF. Caudal regression syndrome verses sirenomelia: A case report. J Perinatol. 2002;22:168-70. [PubMed]

22. Reddy KR, Srinivas S, Kumar S, Redy H, Prasad G, Irfan MM, et al. Sirenomelia a rare presentation. J Neonat] Surg. 2012;1:7. [PMC free article] [PubMed]

23. Aslan H, Yanik H, Celikaslan N, Yildirim G, Ceylan Y. Prenatal diagnosis of Caudal regression syndrome: A case report. BMC Pregnancy Childbirth. 2001;1:8. [PMC free article] [PubMed]

24. González-Quintero VH, Tolaymat L, Martin D, Romaguera RL, Rodríguez MM, Izquierdo LA. Sonographic diagnosis of caudal regression in the first trimester of pregnancy. J Ultrasound Med. 2002;21:1175-8. [PubMed

25. Nievelstein et al. MR of caudal regression syndrome: Embryonic Implications. AJNR 1994;15:1021-1029 